\title{
Energiewende - unter DACH und Fach?
}

\author{
Internationale Gemeinschaftstagung \\ der Energietechnischen Gesellschaften \\ von Electrosuisse, VDE und OVE. \\ 53. Fachtagung der OGE Österreichische \\ Gesellschaft für Energietechnik im OVE
}

\author{
J. Vavra
}

Online publiziert am 28. Oktober 2015

(c) Springer Verlag Wien 2015

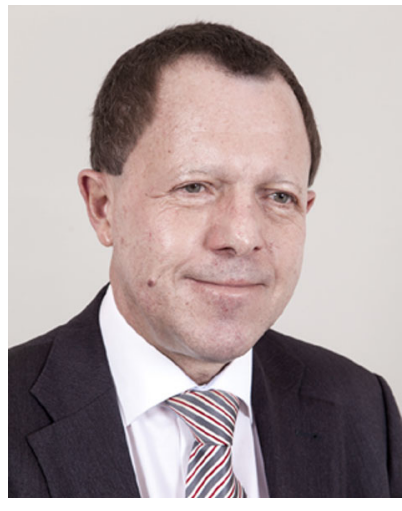

Dipl.-Ing. Johannes Vavra

\begin{abstract}
Sehr geehrte Damen und Herren,

ich begrüße Sie sehr herzlich zur 53. Fachtagung der OGE Österreichische Gesellschaft für Energietechnik im OVE, einer Veranstaltung, welche überdies wieder einmal als internationale Gemeinschaftsfachtagung der energietechnischen Gesellschaften des VDE, der Electrosuisse und des OVE stattfindet.
\end{abstract}

Für den Veranstaltungsort fiel die Wahl diesmal auf Eisenstadt, und es ist wohl

ein bemerkenswertes Detail, dass es mehr als 50 Fachtagungen bedurfte, bis die OGE gewissermaßen das Burgenland für diese Veranstaltung entdeckte. Leichter zu eruieren war damit aber der diesjährige thematische Schwerpunkt, hat doch wohl kein anderes österreichisches Bundesland bei der Stromerzeugung einen derart radikalen Wandel vollzogen wie das Burgenland.

Der Titel unserer diesjährigen OGE-Fachtagung lautet „Energiewende - unter DACH und Fach?", und die Tagung wird sich nicht nur den wesentlichen Fragestellungen widmen, welche sich aus dieser Energiewende ergeben, sondern vor allem auch eine Reihe von möglichen Antworten präsentieren.

Hochkarätige Referenten aus Elektrizitätswirtschaft und Wissenschaft sowie Industrie und Behörden stehen an diesen beiden Tagen zur Verfügung, um die Herausforderungen darzustellen und entsprechende Lösungsansätze zu beschreiben, die Themen aus unterschiedlichen Blickwinkeln zu beleuchten und umfassend zu diskutieren. Natürlich lade ich auch Sie, werte Teilnehmerinnen und Teilnehmer, dazu ein, sich intensiv an diesen Diskussionen zu beteiligen und Ihre Erfahrungen einzubringen!

Die Einführung in das Thema erfolgt mit einer Bestandsaufnahme zu den aktuellen energiepolitischen und strategischen Rahmenbedingungen auf europäischer und nationaler Ebene samt den maß- geblichen Fördersystemen. Davon ausgehend werden die vielfältigen Herausforderungen, welche sich insbesondere für den Ausbau und die Betriebsführung der Netze ergeben, eingehend betrachtet. Dabei ist es aber das erklärte Ziel, nicht bloß über großteils bekannte Probleme zu diskutieren, sondern dem Anspruch einer Fachtagung zu genügen und daher auch konkrete Antworten in Form geplanter oder bereits realisierter technischer Lösungen zu bieten.

Neben der Integration dezentraler Erzeugungsanlagen sind hier vor allem der notwendige Netzausbau und die neuen Anforderungen an Betrieb und Regelung der Netze als Folge dezentraler Einspeisungen mit deren volatilen Eigenschaften zu erwähnen. Dem Speicher, welcher in unterschiedlichen technischen Ausführungen jedenfalls eine Schlüsseltechnologie der Energiewende bildet, wird im Rahmen der Fachtagung gleichermaßen breiter Raum gewidmet.

Für das Gelingen der Energiewende sind zahlreiche Voraussetzungen zu schaffen - neben klaren gesetzlichen Rahmenbedingungen, geeigneten Fördermechanismen und einer gesicherten Finanzierung ist dies natürlich auch eine stabile Stromversorgung trotz und während dieses radikalen Wandels. Die technischen Lösungsansätze stehen im Mittelpunkt der diesjährigen Fachtagung, denn Versorgungssicherheit war nicht nur während der ersten mehr als 100 Jahre das oberste Gebot der Energiewirtschaft, sondern muss es im Sinn einer funktionierenden Gesellschaft auch weiterhin sein

Abschließend möchte ich darauf hinweisen, dass auch heuer wieder die Verleihung der OGE-Preise, des Österreichs EnergiePreises und des Prof. Werner Rieder-Preises im Rahmen der heutigen Abendveranstaltung erfolgt, welche im „Der Reisinger" am Neufelder See stattfinden wird, einem malerischen See, dessen historische Wurzeln überdies eng mit der Stromerzeugung verknüpft sind.

Ich wünsche allen Teilnehmerinnen und Teilnehmern sowie den Referenten eine interessante, qualitativ hochwertige Veranstaltung, darüber hinaus eine informative Exkursion zu einem der drei angebotenen Ziele und darf Sie damit auf der OGE-Fachtagung 2015 herzlich willkommen heißen!

Dipl.-Ing. Johannes Vavra Vorstandsvorsitzender der OGE Österreichische Gesellschaft für Energietechnik im OVE, Wiener Netze GmbH

Vavra, Johannes, Wiener Netze GmbH, Mariannengasse 4-6, 1090 Wien, Österreich (E-Mail: johannes.vavra@wienernetze.at) 